\title{
The role of the post-anaesthesia care unit in the management of high-risk obstetric patients
}

\author{
Georgia Kostopanagiotou ${ }^{1}$, Konstantinos Kalimeris ${ }^{1}$, Aggeliki Pandazi ${ }^{1}$, George Salamalekis², \\ Charalampos Chrelias ${ }^{2}$, Paraskevi Matsota ${ }^{1}$
}

12 $2^{\text {nd }}$ Department of Anaesthesiology, University of Athens School of Medicine,
Attikon Hospital, Athens, Greece
23rd Department of Obstetrics and Gynaecology, University of Athens School of Medicine,
Attikon Hospital, Athens, Greece

Submitted: 17 March 2010

Accepted: 18 June 2010

Arch Med Sci 2011; 7, 1: 123-126

DOI: 10.5114/aoms.2011.20616

Copyright (c) 2011 Termedia \& Banach

\section{Abstract}

Introduction: High-risk obstetric patients in the immediate postpartum period are frequently admitted to the intensive care unit, but the necessity of this practice has recently been doubted. Herein we describe the efficiency of utilizing the post-anaesthesia care unit (PACU) as an intermediate intensive care facility for those patients.

Material and methods: We retrospectively described the reasons for admission, duration of stay, the anaesthetic used, main interventions and outcome for all obstetric admissions in the PACU during a period of 4 years in a university hospital.

Results: During the 4-year period 47 women were admitted to the PACU after delivery. The frequency of admission to the PACU was 15.3 per 1000 deliveries, while obstetric cases represented 4.4 per 1000 admissions to the PACU. The majority represented caesarean sections (81\%). The main reasons for admission to the PACU were haemorrhage (49\%), cardiovascular problems (19\%) and preeclampsia/eclampsia (17\%). Mean length of stay in the PACU was $14.5 \pm 11.6 \mathrm{~h}$, being significantly less in women having received epidural anaesthesia $(8.2 \pm 5.6 \mathrm{~h})$ compared to those who delivered with general anaesthesia (19.0 $\pm 13.6 \mathrm{~h}, p<0.05)$. General anaesthesia was used in $85 \%$ of cases in which emergency delivery was indicated, but only in $27 \%$ of cases without emergency indications for delivery $(p<0.01)$. No death or admission to the intensive care unit occurred during the study period.

Conclusions: The PACU can offer an intermediate intensive care facility for highrisk obstetric patients, thus reducing unnecessary admissions to the intensive care unit.

Key words: maternal morbidity and mortality, complications of pregnancy.

\section{Introduction}

Although postpartum critically ill obstetric patients are often admitted to the intensive care unit (ICU), the necessity of this practice is doubted [1]. Instead, the high-dependency unit has emerged as an alternative facility $[2,3]$. However, establishing such a unit in a hospital where intensive care facilities already exist may also prove unnecessary [4]. In view of the increasing need for intensive care, optimal utilization of intensive care facilities should be pursued.

\author{
Corresponding author: \\ Paraskevi Matsota MD, PhD \\ $2^{\text {nd }}$ Department of \\ Anaesthesiology \\ University of Athens \\ Attikon Hospital \\ 1 Rimini St, Chaidari \\ Postal Code 12462 \\ Athens, Greece \\ Phone: +30 2105015827 \\ Fax: +30 2105326413 \\ E-mail: matsota@yahoo.gr
}


The post-anaesthesia care unit (PACU) may represent a suitable alternative to the high dependency unit, since the characteristics of highrisk postpartum obstetric patients usually allow early recovery if optimal monitoring and medical treatment are readily applied [1]. Advantages of such a strategy could be establishing standard procedures and a possible financial benefit. To this end, we retrospectively described for the first time the utilization of our PACU in treating high-risk obstetric patients in the immediate postpartum period.

\section{Material and methods}

The study was conducted in the University "Attikon" Hospital of the Athens School of Medicine. The PACU consists of 13 beds and a senior anaesthesiologist is in charge, while the nursing personnel is in a $1: 2-3$ ratio to patients. The PACU is staffed overnight and is situated in front of the operating rooms (9), while the obstetric ward is one floor upstairs, consisting of 4 delivery and 2 caesarean section rooms. Inside the maternal ward there is an obstetric recovery room, consisting of 4 beds and used only for short intermediate stay. Our practice is that the clinical director and the senior obstetric anaesthesiologist identify high-risk postoperative obstetric patients and direct them to the PACU. Since official guidelines for intensive care of obstetric patients are lacking, the criteria we used for PACU admission are those of Zeeman et al. and include severe or poorly controlled gestational hypertension, peripartum hysterectomy, major haemorrhage, anaesthetic complications, and severe comorbidities [5]. Consequently, admissions can be either planned or unplanned. We reviewed the medical records of all women admitted to the PACU from the obstetric ward after giving birth, during a period of 4 years (1 January 2005 31 December 2008). Pregnant women operated on for other reasons and deliveries with gestational

Table I. Reasons for admission to the PACU

\begin{tabular}{|lr|}
\hline Reason for admission & $n(\%)$ \\
\hline Haemorrhage & $23(48.9)$ \\
\hline Cardiovascular disorders & $9(19.1)$ \\
\hline Pre-eclampsia/eclampsia & $8(17.0)$ \\
\hline $\begin{array}{l}\text { Haematological disorders (homozygous } \\
\text { sickle cell disease, beta thalassaemia } \\
\text { intermedia and systemic lupus erythematosus } \\
\text { with thrombocytopenia) }\end{array}$ & $3(6.4)$ \\
\hline Neurological disorders & $1(2.1)$ \\
\hline Airway oedema & $1(2.1)$ \\
\hline Liver dysfunction (onset of HELLP) & $1(2.1)$ \\
\hline Pre-existing renal insufficiency (lupus nephritis) & $1(2.1)$ \\
\hline
\end{tabular}

age under 24 weeks were excluded. Data included demographics, gestational age, history of medical problems, mode and urgency of delivery, anaesthetic used, medications, reason for admission, planned or emergency admission to the PACU, therapeutic interventions, PACU stay and maternal outcome. The acute physiology and chronic health evaluation (APACHE II) scores were calculated using the first retrieved data upon arrival in the PACU. The present study is a retrospective analysis, without case control subjects, since all obstetric patients deemed high-risk were admitted to the PACU. Statistical analysis was performed with the Statistical Package for Social Sciences (SPSS) v.15 software. One-way ANOVA was used for comparisons of PACU stay and frequencies of emergency delivery between groups of anaesthesia. Data are presented as mean \pm standard deviation.

\section{Results}

Of the total 3071 women who gave birth, $47(1.53 \%)$ were transferred to the PACU. Total admissions to the PACU were 10766 , representing general surgery, neurosurgery, orthopaedic, gynaecological, head and neck, and vascular surgery patients. Obstetric cases represented $0.44 \%$ of all PACU admissions. Mean maternal age was $30 \pm 6$ years and mean gestational age was $37 \pm 2$ weeks. Mean APACHE II score was $4 \pm 2$, while $38.3 \%$ of admissions had no coexisting disease and $64 \%$ were not on any medication (apart from vitamins and trace elements). Eight women (17\%) had had vaginal delivery and one (2.1\%) had an instrumented vaginal delivery, while the vast majority (81\%) had undergone a caesarean section. Anaesthesia was epidural (EA) in $26 \%$, combined spinal and epidural anaesthesia (CSEA) in $21 \%$, general anaesthesia (GA) in 38\% and both types of anaesthesia in $13 \%$ of women (either due to failed regional anaesthesia or subsequent GA for hysterectomy or abrasion). GA was used in $85 \%$ of emergency delivery cases (11/13), but only in $27 \%$ of cases (9/33) without emergency indications for delivery $(p<0.01)$. Overall, delivery was urgent significantly less frequently in patients who received regional anaesthesia (RA) compared to GA $(7.7 \%$ vs. $55 \%$, respectively, $p<0.01$ ).

Of the 47 admissions $57 \%$ were urgent, while $43 \%$ were planned. Surgical complications were identified in 24 women (48.9\%) (major haemorrhage $29.8 \%$, obstetric hysterectomy $8.5 \%$, obstetric abrasion $6.4 \%$ and re-operation for haemostasis $4.3 \%$ ). The leading reason for admission was haemorrhage $(48.9 \%)$, followed by cardiovascular problems (19\%) and pre-eclampsia/ eclampsia (17\%) (Table I). Cardiovascular problems included valvular heart disease (5 patients), chronic heart failure (3 patients) and tachyarrhythmia of 
acute onset (one patient). Non-prophylactic medications were used in $34 \%$ of women, including intravenous antihypertensives (12.7\%), diuretics (8.5\%) and corticosteroids (8.5\%). Blood transfusion was performed in $44.8 \%$ of women, while fresh frozen plasma and platelets were transfused in 19.1\% (Table II). Mechanical ventilation was used only in one patient until she was stabilized and successfully extubated. Arterial pressure was invasively monitored in all cases and pulmonary artery pressures were monitored in 2 patients (severe mitral valve stenosis). An intrauterine balloon was placed in one patient with significant thrombocytopenia and ongoing uterine haemorrhage, after unsuccessful re-operation for haemostasis, resulting in avoidance of hysterectomy. No maternal death, neurological disorder or deterioration of other pre-existing organ insufficiency was observed.

No patient was transferred to the ICU. Mean length of stay in the PACU was $15 \pm 12 \mathrm{~h}$. The length of PACU stay was $16 \pm 13 \mathrm{~h}$ for patients who received CSEA, $19 \pm 14 \mathrm{~h}$ for GA, $10 \pm 7 \mathrm{~h}$ for RA converted to $\mathrm{GA}$ and $8 \pm 6 \mathrm{~h}$ for EA. The difference in PACU stay was significant between groups of EA and GA ( $p=0.012$ ). PACU stay was $12 \pm 11 \mathrm{~h}$ for planned admissions and $16 \pm 12 \mathrm{~h}$ for urgent admissions $(p>0.05)$. There was also no difference in PACU stay between patients who received blood transfusions $(17 \pm 14 \mathrm{~h})$ and those who did not $(13 \pm 10 \mathrm{~h})$.

\section{Discussion}

Our experience in utilizing the PACU for postpartum treatment of high-risk obstetric patients is encouraging in that it prevented all ICU admissions and was associated with a short stay $(<24 \mathrm{~h})$. Literature on obstetric PACU admissions is lacking, while high-risk obstetric patients in nonprimarily obstetric hospitals are usually handled by the ICU. The obstetric admission ratio found here is increased compared to respective data on ICU admissions $[1,4,6]$. This may be associated with the referral of those patients to tertiary facilities, or with the change of current practice towards closer management. However, the obstetric admission rate is comparable with reported data on obstetric utilization of the ICU $[3,5,7]$. Maternal mortality was not noted in our study; however, the population studied is obviously insufficient to address this issue. The mean APACHE II score was low, reflecting the fact that most patients were young and otherwise healthy $[7,8]$.

The most frequent reason for admission was major haemorrhage, followed by preeclampsia/ eclampsia. Although obstetric haemorrhage is among the leading causes for ICU admission, the systemic use of oxytocin, appropriate fluid replacement, blood transfusions, correction of
Table II. Blood product transfusions in the postanaesthesia care unit

\begin{tabular}{|lc|}
\hline Transfusion & $n(\%)$ \\
\hline No transfusion & $26(55.3)$ \\
\hline $1-2 \mathrm{U}$ pRBC & $14(29.8)$ \\
\hline $3-6 \mathrm{U}$ pRBC & $6(12.8)$ \\
\hline$>6$ U pRBC & $1(2.1)$ \\
\hline FFP and/or PLT & $9(19.1)$ \\
\hline
\end{tabular}

pRBC - packed red blood cells, FFP - fresh frozen plasma, PLT - platelets

coagulopathies and early surgical intervention possibly contributed to early discharge from the PACU $[1,9,10]$. Despite these measures, hysterectomy was deemed necessary in 3 patients. Patients with preeclampsia/eclampsia were treated with intravenous (hydralazine, nitroglycerine) or oral (methyldopa) antihypertensives, while care was taken for maintaining diuresis and anticonvulsive therapy with magnesium sulphate. Reasons for admission are comparable with published data on ICU obstetric admissions, except for respiratory complications, namely pulmonary embolism, which usually occur in the later recovery period $[6,11]$.

The mean stay in the PACU was relatively short, being shorter in women having received epidural anaesthesia. Managing acute complications and preventing exacerbations of underlying diseases probably contributed to the short stay [4]. Obstetric RA is routinely used in our department, while GA is the choice in emergencies or when RA is contraindicated or refused by the patient. The shorter stay with epidural anaesthesia probably reflects the less frequent urgency for delivery as observed here, rather than a pure advantage of RA. Indeed, GA is an independent risk factor for ICU admission, as it is dictated in emergency caesarean section, and it is also preferred in coagulopathies and anticipated major haemorrhage $[6,12]$. On the other hand, RA is preferred with expected difficult airway $[13,14]$. However, RA has never been conclusively shown to reduce maternal mortality and an obvious bias can be introduced against GA when comparing patient outcomes.

The nursing personnel in our PACU is familiarized with both intensive care and obstetric nursing, substantially facilitating this approach. Although this may require demanding staff training, our experience is encouraging. Moreover, patient volume did not affect bed availability in the PACU or medical and nursing staff requirements. Taking into account the very low admission rate of highrisk obstetric patients in the PACU, our opinion is that the de novo establishment of a highdependency unit for these patients in our hospital would result in unacceptable health costs. However, in hospitals without a PACU available, a highdependency unit would be of great value in 
accommodating such patients, reducing the need for ICU admission. It should also be noted that all patients were admitted to the PACU according to the criteria of Zeeman et al. [5], which identify obstetric patients who should preferentially receive intensive care in critical care facilities instead of standard care in the ward. Further advantages are direct supervision by the assigned anaesthesiologist and appropriate obstetric consultation. In our opinion, the prevention of some urgent admissions to the PACU and the reduction of complications and need for prolonged intensive care is largely based on the timely response of the obstetric, anaesthesiological and blood bank teams. This should be best accomplished by familiar protocols that are regularly practised by the personnel of those teams. Regarding the prevention of planned admissions to the PACU, the close observation of pregnancy, early identification of complications and risk factors (e.g. placenta praevia) and early consultation by specialists could reduce some admissions of such patients, since the major reasons for PACU admissions are haemorrhage, uncontrolled hypertension and cardiovascular complications. It is more preferable to closely monitor for a few hours a high-risk obstetric patient with an uneventful delivery rather than manage complications that could have been prevented. Nevertheless, patients with failure of two or more organs, or with predicted stay $>24 \mathrm{~h}$, would be better transferred to the ICU.

The post-anaesthesia care unit was successfully used as an intermediate facility to treat high-risk postpartum obstetric patients, avoiding unnecessary ICU admissions. The ideal management of these patients should if possible be scheduled based on the individual features of a given institution. Where no PACU or ICU is available in the proximity, a high-dependency unit would be undoubtedly useful. In a non-primarily obstetric hospital such as ours, taking advantage of the PACU as an intermediate facility seems a satisfactory alternative. Although this policy should not reduce the need for ICU admission for the most severe cases, a controlled study would clarify any possible advantages of PACU usage on patient outcome and health costs.

\section{References}

1. Mirghani HM, Hamed M, Ezimokhai M, Weerasinghe DS. Pregnancy-related admissions to the intensive care unit. Int J Obstet Anesth 2004; 13: 82-5.

2. Saravanakumar K, Davies L, Lewis M, Cooper GM. High dependency care in an obstetric setting in the UK. Anaesthesia 2008; 63: 1081-6.

3. Ryan M, Hamilton V, Bowen M, McKenna P. The role of high-dependency unit in a regional obstetric hospital. Anaesthesia 2000; 55: 1155-8.

4. Panchal S, Arria AM, Harris AP. Intensive care utilization during hospital admission for delivery: prevalence, risk factors, and outcomes in a statewide population. Anesthesiology 2000; 92: 1537-44.

5. Zeeman GG, Wendel GD, Cunningham FG. A blueprint for obstetric critical care. Am J Obstet Gynecol 2003; 188: 532-6.

6. Selo-Ojeme DO, Omosaiye M, Bhattacharjee P, Kadir RA. Risk factors for obstetric admissions to the intensive care unit in a tertiary hospital: a case control study. Arch Gynecol Obstet 2005; 272: 207-21.

7. Koch KA, Rodeffer HD, Sanchez-Ramos L. Critically ill obstetrical patients: outcome and predictability. Crit Care Med 1988; 16: A409.

8. el-Solh AA, Grant BJ. A comparison of severity of illness scoring systems for critically ill obstetric patients. Chest 1996; 110: 1299-304.

9. Al-Zirqi I, Vangen S, Forsen L, Stray-Pedersen B. Prevalence and risk factors of severe obstetric hemorrhage. BJOG 2008; 115: 1265-72.

10. Wise A, Clark V. Strategies to manage major obstetric hemorrhage. Curr Opin Anaesthesiol 2008; 21: 281-7.

11. Al-Suleiman SA, Qutub HO, Rahman J, Rahman MS. Obstetric admissions to the intensive care unit: a 12-year review. Arch Gynecol Obstet 2006; 274: 4-8.

12. Stephens ID. ICU admissions from an obstetrical hospital. Can J Anaesth 1991; 38: 677-81.

13. Dresner MR, Freeman JM. Anaesthesia for caesarean section. Best Pract Res Clin Obstet Gynaecol 2001; 15: 127-43.

14. Eltzschig HK, Lieberman ES, Camann WR. Regional anaesthesia and analgesia for labor and delivery. New Engl J Med 2003; 348: 319-32. 